\title{
BASIC OXIDES ON ACTIVE CARBON
}

\author{
Chanel Ishizaki* and Iris Marti \\ (Received June 18, 1987) \\ Institute Venezolano de Investigaciones \\ Cientificas (I.V.I.C.) \\ Apartado 21827, Caracas 1020-A Venezuela \\ *Analysis Center, Nagaoka University of Technology \\ Nagaoka, Niigata 940-21, Japan \\ (To whome correspondence should be addressed).
}

A direct transmission IR analytical technique capable of distinguishing quali as well as quantitative spectral differences has been applied to a series of active carbons. The amount of acidic and basic groups was also evaluated by standard neutralization techniques. For the carbons with acidic and basic surface properties three broad but distrinctive bands were observed, centred around 1735,1585 and $1240 \mathrm{~cm}^{-1}$ meanwhile a carbon without strong acidic oxides showed only the 1585 and $1240 \mathrm{~cm}^{-1}$ bands. A review of the literature on previously reported IR spectra of several carbonaceous materials including: coals, carbon blacks, graphite, chars and active carbons is presented, discussed and interpreted together with the findings of the present study.

A linear direct correlation was found between the intensities of the 1585 and 1240 $\mathrm{cm}^{-1}$ absorption bands for the carbon which is basic in nature. The results are interpreted as a confirmation of the $r$-pyronic like structures proposed by Boehm for the basic oxides.

KEYWORDS: Active Carbon, Chemical Surface Characteristics, IR Analysis, Basic Oxides

\section{INTRODUCTION}

The surface properties of active carbons and carbon blacks are stongly influenced by the presence of surface oxides which are formed during the manufacturing process. The actual chemical nature of these oxygen containing groups has drawn the attention of a considerable number of investigators since $1863^{1)}$ but up to the present time total agreement about the different functionalities has not been reached. All spectroscopic methods have successfully been applied in this field ${ }^{2}$. Among them IR has certainly found the most frequent appli- cation because of its highly instructive information. IR spectra analysis provides direct evidence of functional groups occurring in the non-modified substances: the absorption bands observed correspond to definite vibration frequencies of certain atomic bonds. In principle the intensity of these bands is also a measure of the number of bonds of a certain type present. The IR method in-situ detects the presence of chemical groups, precludes or limits the existence of proposed structures and demonstrates similarities and differences in chemical structure. The spectra stand as characteristic properties 
that must be explained by any structure proposed.

In a previous publication by the authors ${ }^{3}$ ) some understanding on the nature of the surface functional groups of a commercially available active carbon was obtained combining IR direct transmission spectrocopy with the selective neutralization technique outlined by Boehm ${ }^{4)}$. In the present investigation the stress is on the development of an IR analytical technique capable of measuring quali as well as quantitative differences on a series of active carbons. In order to accomplish this objective the amount of surface functional groups of a reference carbon was varied by heat treatment in an inert atmosphere and by oxidation with oxygen gas. The reason for taking this approach was based on the very well known facts that the heat treatment at elevated temperatures in an inert atmosphere eliminates most of the oxygen functional groups from the surface and that surface oxygen groups can be produced again by oxygen oxidation ${ }^{5}$ ). An excellent review of the literature on the formation and stability of surface oxides in the oxidation of carbon has been presented by Boehm ${ }^{6}$.

\section{EXPERIMENTAL}

A commercial granular active carbon produced from coal by high temperature steam activation (Filtrasorb 200 from Calgon Corporation) was investigated. The mean particle diameter used was $616 \mu \mathrm{m}$. Dust was removed from the carbon by rinsing several times with distilled water. The carbon particles were then oven dried in thin layers at $105^{\circ} \mathrm{C}$ for $24 \mathrm{~h}$ and stored in air tight glass jars. The carbon (A) was subjected to the following treatments. It was heat treated at $1,000^{\circ} \mathrm{C}$ in a nitrogen atmosphere for $17 \mathrm{~h}$ and allowed to cool down to room temperature under the same atomosphere. This carbon will be identified as A-OG. The surface of carbon $\mathrm{A}$ was also modified by heating at $1,000^{\circ} \mathrm{C}$ under nitrogen atomosphere for 17 hours, and allowed to cool to $400^{\circ} \mathrm{C}$ at which nitrogen was displaced by oxygen and the oxidation was accomplished at this temperature for $30 \mathrm{~min}$ with an oxygen flow of $40 \mathrm{ml} / \mathrm{min}$. After the oxidation the carbon was cooled to room temperature under nitrogen atmosphere. This carbon is labeled A-OGOX $400^{\circ} \mathrm{C}$.

All these carbons were subjected to IR investigation using $\mathrm{KBr}$ pellets. The $\mathrm{KBr}$ pellets of the different carbon samples were prepared in the following manner: a given amount of carbon sample was ground for $4 \mathrm{~min}$ in a stainless steel grinding capsule with a vibrating mill. After the addition of $200 \mathrm{mg}$ of $\mathrm{KBr}$ the mixture was ground for an additional half minute and pressed. Extreme precautions were taken to avoid water contamination. Spurious bands are observed due to water contamination ${ }^{7)}$. The spurious bands were eliminated in our case if the pellets were storaged under vacuum in a desiccator with silica gel. The same observation was reported by Robin and Rouxhet ${ }^{8)}$.

IR direct transmission spectra of the dried pellets were obtained using a Perkin-Elmer Model 580 IRS coupled with an Interdata Model 6/16 Computer. The spectra were recorded in a moisture and $\mathrm{CO}_{2}$ - free atmosphere. The analysis was confined to the $1850-1150$ $\mathrm{cm}^{-1}$ region due to the uncertainty of possible water absorption in the $\mathrm{OH}$ stretching region.

The amount of carbon present in the pellets was optimized to be able to obtain the spectra without using attenuation in the reference beam. The working range obtained was up to $0.5 \mathrm{mg}$ for the carbon A-OGOX $400^{\circ} \mathrm{C}$ and up to 0.7 $\mathrm{mg}$ for the other carbons. The amount of carbon in the pellets was varied between 0.2 and $0.5 \mathrm{mg}$. In order to quantify the intensity of the different bands obtained in the spectra each spectrum recorded in percent transmittance using a resolution mode of $2.8 \mathrm{~cm}^{-1}$ was accumulated five times, converted to absrobance and multiplied by a factor of two. The intensity of the areas for each band were then calculated with the aid of the computer.

Standard neutralization techniques with $\mathrm{NaOH}, \mathrm{Na}_{2} \mathrm{CO}_{3}, \mathrm{NaHCO}_{3}$ and $\mathrm{HCl}$ solutions were performed. Approximately $0.2 \mathrm{~g}$ of carbon and $20 \mathrm{ml}$ of $0.25 \mathrm{~N}$ solution was used for each neutralization. The carbons were allowed to react for $24 \mathrm{~h}$ under constant agitation. After this period the carbon was removed from the solution by centrifugation and the solution retitrated. 


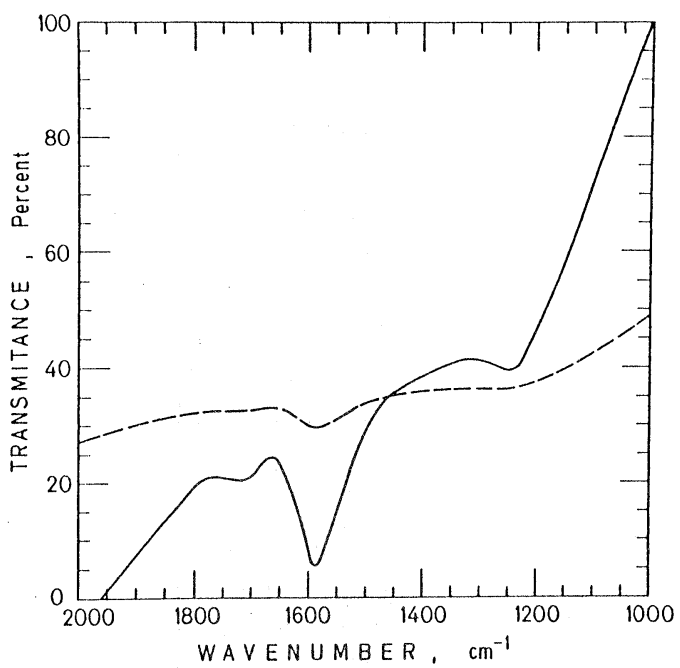

Fig. 1 IR-direct transmission Spectra of carbon A. --- Single spectrum. - Five times accumulated spectrum.

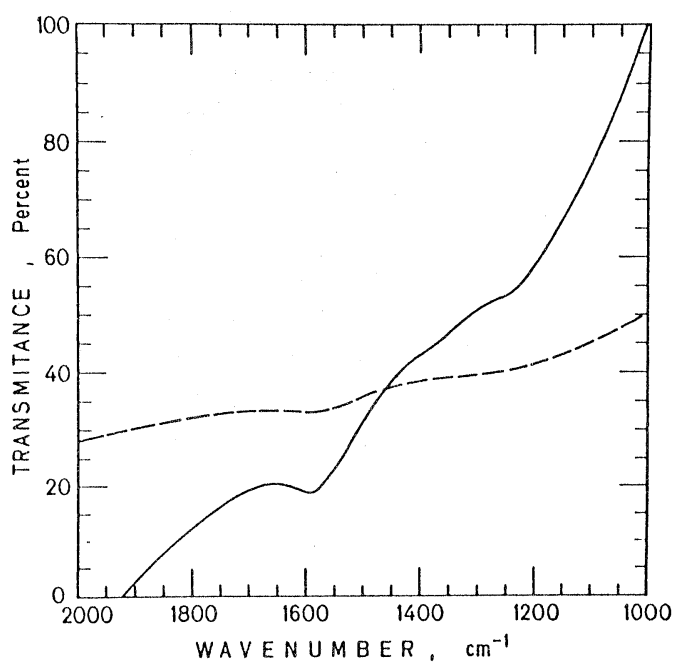

Fig. 2 IR-direct transmission spectra of carbon A-OG. --- Single spectrum. - Five times accumulated spectrum.

\section{RESULTS AND DISCUSSION}

Figures 1-3 show representative spectra for the carbons A, A-OG and A-OGOX $400^{\circ} \mathrm{C}$ respectively. Three broad but distinctive bands centered at 1735,1585 and $1240 \mathrm{~cm}^{-1}$ are observed for the original carbon (A) and the oxidized one (A-OGOX $400^{\circ} \mathrm{C}$ ) meanwhile the heated carbon (A-OG) shows only two

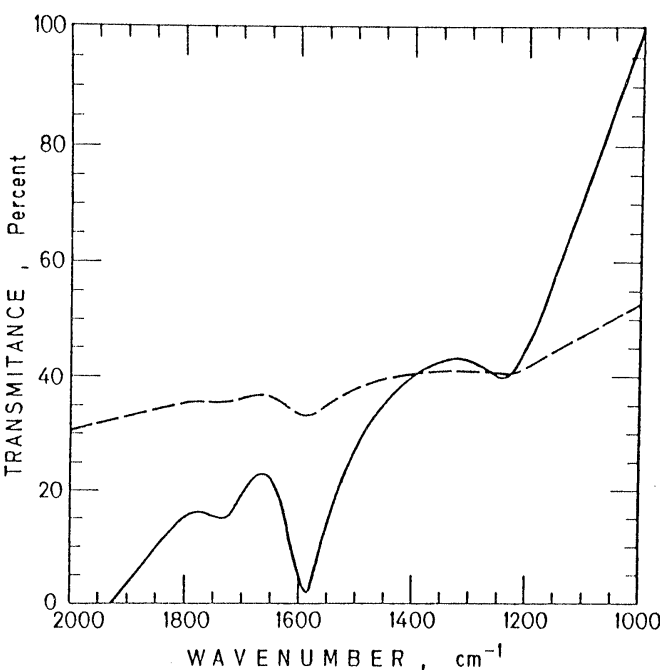

Fig. 3 IR-direct ransmission spectra of carbon A-OGOX $400^{\circ} \mathrm{C}$. -- Single spectrum. - Five times accumulated spectrum.

absorption bands, lacking the band centered at $1735 \mathrm{~cm}^{-1}$. Table 1 shows the results of the neutralization experiments which indicate as expected that most of the acidic groups have been removed from carbon A-OG and have been formed again by oxidation as shown by the A-OGOX $400^{\circ} \mathrm{C}$ carbon. At the same time the spectra show that the $1735 \mathrm{~cm}^{-1}$ band, which corresponds to acidic carbonyl vibrations reappears after the A-OG carbon is oxidized at $400^{\circ} \mathrm{C}$ with oxygen gas. The nature of the acidic functional groups was discussed earlier ${ }^{3}$ ) and will not be discussed here. In the present work the stress will be on the basic oxides.

Table 1 Neutralization Capacities for Different Carbons.

\begin{tabular}{lccc}
\hline $\begin{array}{c}\text { Neutralizing } \\
\begin{array}{c}\text { Solution } \\
(0.25 \mathrm{~N})\end{array}\end{array}$ & $\begin{array}{c}\text { Amount Neutralized by } \\
\text { Different Carbons in } \\
\text { meq/100g }\end{array}$ \\
\hline & $\mathrm{A}$ & A-OG & A-OG400 ${ }^{\circ} \mathrm{C}$ \\
\cline { 2 - 3 } & $74 \pm 8$ & $7 \pm 6$ & $137 \pm 7$ \\
$\mathrm{NaOH}$ & $26 \pm 18$ & 0 & $63 \pm 13$ \\
$\mathrm{Na}_{2} \mathrm{CO}_{3}$ & $7 \pm 0$ & 0 & $36 \pm 19$ \\
$\mathrm{NaHCO}_{3}$ & $50 \pm 12$ & $52 \pm 14$ & $33 \pm 14$ \\
$\mathrm{HCl}$ & &
\end{tabular}


Fiedel and Hofer $\left.{ }^{9}\right)$ claim to have obtained the first direct transmission IR spectra of an active carbon. These authors also used $\mathrm{KBr}$ pellets and found three distinctive bands centered around 1735,1590 and $1212 \mathrm{~cm}^{-1}$. Since the functionality of active carbons and carbon blacks is rather similar it may also be instructive to compare their IR spectra. Garten et al. ${ }^{10}$ ) reported two absorption bands in the 2000 $1500 \mathrm{~cm}^{-1}$ region for a carbon black, at 1760 and $1600 \mathrm{~cm}^{-1}$. Studebaker and Rinehart ${ }^{11}$ ) also obtained IR spectra of carbon blacks in the $2000-1000 \mathrm{~cm}^{-1}$ region and reported bands centered in the following regions, $1775-1675$, $1675-1550$ and $1275-1195 \mathrm{~cm}^{-1}$. Prest and Mosler ${ }^{12)}$ using FTIR spectroscopy to investigate the functional groups on carbon blacks also found three distinctive peaks with maxima at 1725,1595 and $1245 \mathrm{~cm}^{-1}$. Starsinic et al. ${ }^{13}$ ) applied FTIR to the study of oxidized Saran chars and also found three distinctive bands centered at 1720,1585 and $1250 \mathrm{~cm}^{-1}$ respectively.

The review of the literature as well as the results of the present work clearly indicate that the reported direct transmission IR spectra of active carbons as well as carbon blacks show similar patterns, indicating very distinctive and reproducible qualitative features. The main differences among the researchers that have studied the functional groups on different carbonaceous materials by IR spectroscopy lay on the interpretation of the observed bands. The most controversial assignment for the observed bands in a series of carbonaceous materials: coals ${ }^{14)-25)}$, carbon blacks ${ }^{10)-12)}$,

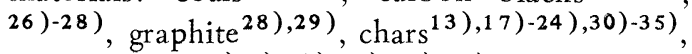
active carbons $\left.\left.\left.\left.\left.{ }^{3}, 9\right), 10\right), 28\right), 35\right), 36\right)$, has been the $1600 \mathrm{~cm}^{-1}$ band. This band has been assigned to the aromatic structure, hydrogen bonded carbonyl groups, quinone structures and thermally stable carboxyl-carbonate structures. It is generally agreed that the absorption in this region is too intense to be attributed solely to aromatic ring vibrations. It has been suggested that the intensity may be largely due to the aromatic ring vibrations enhanced by polar (oxygenated) substituents or to direct contribution by conjugatively chelated carbonyl groups.
An effort was made by Friedel and Retcofsky ${ }^{25)}$ to search the nuclear magnetic resonance spectrum for the possible existence of the conjugated, chelated carbonyl structure.

$$
\mathrm{OH}-\ldots-\mathrm{O}=\mathrm{C}-\mathrm{C}=\mathrm{C}-
$$

Their results established that appreciable numbers of strongly chelated protons are not present in the same structure that shows intense IR absorption in the $1600 \mathrm{~cm}^{-1}$ region. The authors gave a possible explanation for this band in coals as metal derivates of chelated, conjugated carbonyl structures.

$$
-\mathrm{O}-\mathrm{METAL}-\mathrm{O}=\mathrm{C}-\mathrm{C}=\mathrm{C}-
$$

Friedel et al. ${ }^{30)}$ using oxygen -18 labeled chars failed to obtain any appreciable shift in the $1600 \mathrm{~cm}^{-1}$ band due to the isotope effect and considered that this observation appeared to suggest that oxygen containing groups may not be contributing, either directly or indirectly to the intensity of the $1600 \mathrm{~cm}^{-1}$ band. On the other hand Friedel and coworkers ${ }^{17), 24)}$ had shown that chars prepared from carbohydrates $\left(\leq 500^{\circ} \mathrm{C}\right.$ ) invariably exhibit coal like spectra with an intense absorption feature near 1600 $\mathrm{cm}^{-1}$, and that chars prepared from other oxygenated compounds as well as hydrocarbons chared in presence of oxygen often presented coal like spectra. This apparent contradiction was solved by Friedel after obtaining the IR spectra of graphite ${ }^{29}$ ) reassigning the $1600 \mathrm{~cm}^{-1}$ band in coals and chars to graphitic structures. As a major assignment this may be correct but the enhancement of the band due to oxygen chemisorption has been shown by Zawad$\mathrm{sky}^{31), 32)}$, in a very convincing manner, and recently Starsinic et al. ${ }^{13)}$ also arrived to the same conclusion.

The results of Starsinic et al., also ruled out the assignment of the $1585 \mathrm{~cm}^{-1}$ band to highly conjugated hydrogen bonded carbonyl groups. On the other hand the results presented by Studebaker and Rinehard ${ }^{11}$ ) show that the assignment made by them to the $1600 \mathrm{~cm}^{-1}$ band, as quinone groups is supported by the good correlation found between the integrated intensity across the $1675-1550 \mathrm{~cm}^{-1}$ and the quinone content from reduction with $\mathrm{NaBH}_{4}$.

In order to obtain a better insight of the correspondence between the observed bands in relation to the surface groups functionalities 
a systematic quantification of band intensities was undertaken in the present study. Figure 4 shows a typical five times accumulated spectrum in transmittance and the corresponding spectrum in absorbance times a factor of two. The three bands are indicated as well as the corresponding areas. The initial and final wave numbers taken for each band in computing the areas were as follows: $A_{1}: 1770-1650 \mathrm{~cm}^{-1}, A_{2}: 1670-1400$ $\mathrm{cm}^{-1}$ and $A_{3}: 1370-1190$ (minimum) and 1150 $\mathrm{cm}^{-1}$ (maximum). By changing the amount of carbon present in the pellet different band intensities were obtained. The areas of the different bands were calculated with the aid of the computer as stated in the experimental section. For each particular spectrum the area of each band was divided by the amount of carbon in the pellet.

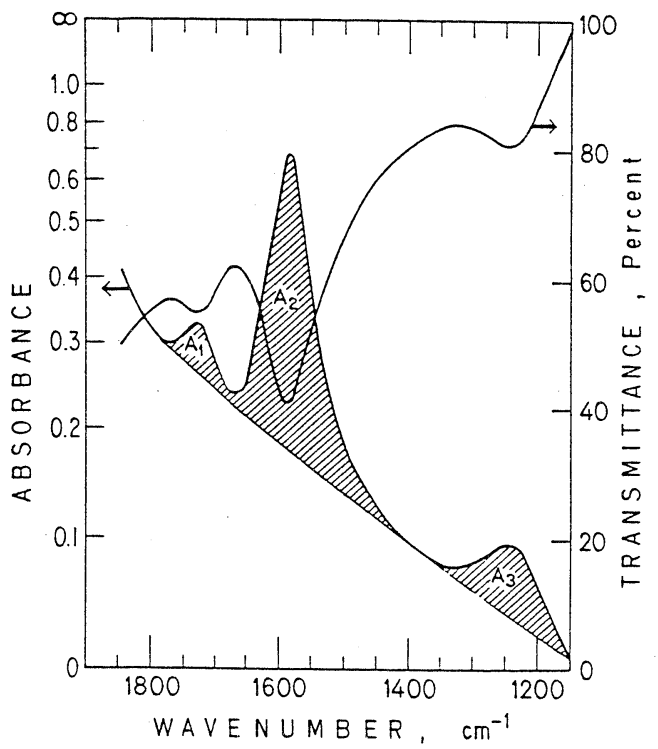

Fig. 4 IR-direct transmission spectrum showing a five times accumulted spectrum in $\%$ transmission and the resultant spectrum after conversion into absorbance and multiplication by a factor of two.

As stated earlier the carbon which was heated in nitrogen atmosphere at $1,000^{\circ} \mathrm{C}$ (A-OG) did not show the presence of the strong acidic groups indicated by the absence of the 1735 band $\left(A_{1}\right)$ (Fig. 2). For this carbon a linear direct correlation was found between the in- tensity of the absorbance of the $A_{2}$ band per unit weight of carbon $\left(B_{2}\right)$ and the corresponding intensity of the $A_{3}$ band per unit weight of carbon $\left(B_{3}\right)$ as shown in Fig. 5. The equation that represents this correlation is:

$$
\mathrm{B}_{3}=0.94( \pm 0.67)+0.20( \pm 0.05) \mathrm{B}_{2} \text { (Eq. 1) }
$$
with a correlation coefficient equal to one. For this particular carbon the intensity of the $A_{1}$ band is equal to zero and therefore $B_{1}$ is also zero.

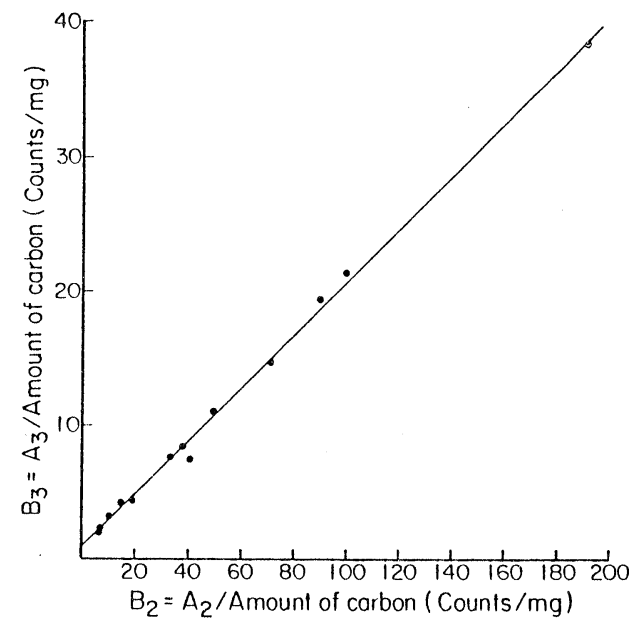

Fig. 5 Correlation between the intensity of the $\mathrm{A}_{3}$ band per unit weight of carbon $\left(B_{3}\right)$ and the intensity of the $A_{2}$ band per unit weight of carbon $\left(B_{2}\right)$ for carbon A-OG.

Prest and Mosler ${ }^{12)}$ even though did not undertake a systematic measurement of the areas, also suggested a very good correspondence between the 1590 and $1245 \mathrm{~cm}^{-1}$ bands.

Since the acidic nature of the A-OG carbon is negligible (Table 1) it may be considered that the observed absorption bands correspond to basic oxides. Based on chemical evidence, Voll and Boehm ${ }^{36}$ ) proposed a pyrone like structure for the basic oxides with the two oxygen atoms located in different rings of a graphitic layer as shown in Fig. 6.

The good linear correlation found between the intensities of the $1670-1400 \mathrm{~cm}^{-1}$ and the $1370-1190 \mathrm{~cm}^{-1}$ bands for the A-OG carbon can be explained by structures similar to the ones shown in Fig. 6 in the following manner. The ether linkages will give absorption in the 


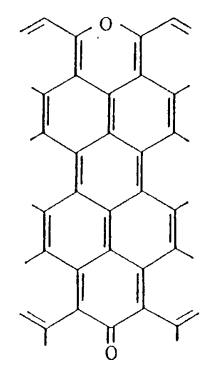

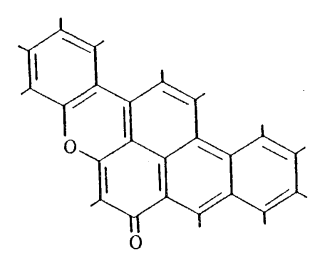

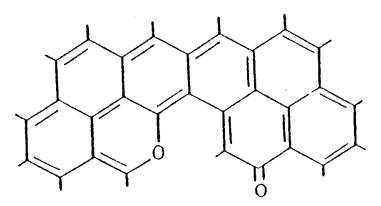

Fig. 6 Basic oxide structures proposed by Voll and Boehm ${ }^{36)}$.

$1370-1190 \mathrm{~cm}^{-1}$ range, and the carbonyl plus the graphitic structure enhanced by the oxygen substituents will give corresponding overlapping absorptions in the $1670-1400 \mathrm{~cm}^{-1}$ range. The quantity of ether linkages and carbonyl groups appears to have a systematic array as expected and therefore the absorption intensities correlate for each given amount of carbon sample.

This type of structure will also explain the enhancement of the aromatic ring vibrations. As Bellamy ${ }^{38}$ ) indicates the $1600 \mathrm{~cm}^{-1}$ doublet arises from vibrations in which the main dipole moment change is produced by the movements of the substituents on opposite sides of the ring acting in opposition. With polar substituents the change is larger and the band intensifies, and this occurs independently of whether the substituent is an electron acceptor or a donor.

Furthermore, Papirer et al. ${ }^{39)}$ have recentely suggested a mechanism for the basic oxides formation on carbon blacks which supports the structures proposed by Voll and Boehm ${ }^{36)}$ and is also in agreement with the results of the present study. The mechanism proposed by Papirer et al. indicates that pyrone-type structures are generated by thermal decomposition of oxygenated acidic groups, formation of active sites capable to fix oxygen in an ether form and rearrangement with existing carbonyl groups which resist pyrolysis.

The A-OG carbon used in our study was the heat treated product of a carbon which previously had acidic groups as shown in Table 1 , and Fig. 1. The heat treatment was carried out in an inert atmosphere and cooled down under the same atmosphere to eliminate and avoid the formation of acidic oxides. The exposure to oxygen after the treatment was done at room temperature. The conditions for the preparation of the A-OG carbon of our study were similar to those of Papirer et al. and therefore it is very likely that the same kind of mechanism occurred and a similar end product was obtained.

\section{CONCLUSIONS}

The present study indicates that an active carbon which exhibits only a basic nature presents only two broad IR bands centered around $1670-1400 \mathrm{~cm}^{-1}$ and $1370-1190 \mathrm{~cm}^{-1}$. By changing the amount of carbon present in the $\mathrm{KBr}$ pellets (between 0.2 and $0.5 \mathrm{mg}$ carbon in $200 \mathrm{mg} \mathrm{KBr}$ ) a linear correlation was found between the intensities of the two bands. The results are interpreted as a confirmation of the pyrone type groups proposed by Voll and Boehm since for these particular structures the ether linkages which would give rise to the absorption intensities in the range $1370-1190 \mathrm{~cm}^{-1}$ should be in correspondence with the carbonyl group which would show the bands in the 1670-1400 $\mathrm{cm}^{-1}$ range plus the corresponding intensification of the aromatic ring vibration due to the oxygen substituents in the same range. The results of the present study are also in agreement with the basic oxides formation mechanism recently proposed by Papirer et al.

\section{ACKNOWLEDGMENTS}

Part of this work was perfomed during Dr. Ishizaki's sabbatical leave spent at the Swiss Federal Institute for Water Resources and Water Pollution Control (EAMAG).

The intellectual assistance given by the members of the Chemistry Department, as well as the financial support are greatly appreciated.

Dr. Ishizaki is in debted to Dr. K. Mutter, head of the Organic Chemistry Group of the Swiss Federal Laboratories for Material Testing and Research (EMPA) for allowing to use the IR Spectrophotometer and Data System, and useful conversations. Special recognition is conferred to Mrs. I. Farrenkothen, head of the 
Gaschromatography and Infra-red Spectroscopy Section of the Organic Chemistry Group of EMPA for the professional guidance, friendship and moral support given during the development of the analytical method.

\section{LITERATURE CITED}

1) R.A. Smith, Proc. Roy. Soc. (London) 12, $424(1863)$

2) H. Knozinger, Acta Cientifica Venezolana 24 Suplemento 2, 76 (1973)

3) C. Ishizaki and I. Marti, Carbon 19, 409 (1981)

4) H.P. Boehm, In "Advances in Catalysis", D.D. Eley, M. Pines, P.B. Weisz Eds. Academic Press, New York, Vol. 16, (1966) p. 179

5) B.R. Puri, In "Chemistry and Physics of Carbon", P.L. Walker, Jr. Ed., Marcel Dekker, New York, Vol. 6, (1970) p. 191

6) H.P. Boehm and G. Bewer, In "Proceedings 4th Conference Industrial Carbon and Graphite", Soc. Chem. Industry, London (1974) p. 344

7) R.A. Friedel, In "Applied Infra-red Spectroscopy", D.N. Kendall, Ed., Reinhold Pub. Corp., New York (1966) p. 312

8) P.L. Robin and P.G. Rouxhet, Fuel 55, 177 (1976)

9) R.H. Friedel and L.J.E. Hofer, J. Phys. Chem. 74, 2921 (1970)

10) V.A. Garten, D.E. Weiss and J.B. Willis, Aust. J. Chem. 10, 295 (1957)

11) M.L. Studebaker and R.W. Rinehard, Rubber Chem. Technol. 45, 106 (1972)

12) W.H. Prest Jr. and R.A. Mosler, In "Colloids and Surfaces in Reprographic Technologies", M. Hair, M. Croucher Eds.; A.C.S. Symposium Series: Washington D.C., Chapter 12 (1982)

13) M. Starsinic, R.L. Taylor, P.L. Walker, Jr. and P.C. Painter, Carbon 21, 69 (1983)

14) C. Cannon and B. Sutherland, Trans. Farad. Soc. 41, 279 (1945)

15) R.R. Gordon, W.N. Adams and G.J. Jenkis, Nature 170, 317 (1952)

16) C.G. Cannon, Nature 171, 308 (1953)

17) R.A. Friedel and M.G. J, Pelipetz, Opt. Soc.
Am. 43, 1051 (1953)

18) R.R. Gordon, W.N. Adams, G.J. Pitt and G.H. Watson, Nature 174, 1098 (1954)

19) H.A. van Vucht, B.J. Rietveld and D.W. van Krevelen, Fuel 34, 50 (1955)

20) J.K. Brown and W.E. Wyss, Chem. and Ind. 1955, p. 1118

21) J.K. Brown, Chem. Soc. 1955, p. 744

22) R.A. Friedel and J.A. Queiser, Anal. Chem. 28, 22 (1956)

23) J.D. Brooks, R.A. Durie and S. Sternhell, Aust. J. Appl. Sci. 9, 63 (1958)

24) R.A. Friedel, In "Proceedings of the Fourth Conference on Carbon", Pergamon Press; Oxford. Vol. 2, (1960) p. 321

25) R.A. Friedel and H. Retcofsky, In "Proceedings of the Fifth Conference on Carbon", Pergamon Press; Oxford, Vol. 2 (1963) p. 149

26) R.A. Friedel and G.L. Carlson, Fuel 51, 194 (1972)

27) V.J. Lygin, N.V. Kovalera, N.N. Kantaradze and A.V. Kiselev, Kolloidn Zurnal 22, 334 (1960)

28) J.S. Mattson and H.B. Mark. Jr., J. Colloid Interface Sci. 31, 131 (1969)

29) R.A. Friedel and G.L. Carlson, J. Phys. Chem. 75, 1149 (1971)

30) R.A. Friedel, R.A. Durie and Y. Shewchyk, Carbon 5, 559 (1967)

31) J. Zawadski, Carbon 16, 491 (1978)

32) J. Zawadski, Polish J. of Chem. 52, 2157 (1978)

33) J. Zawadski, Carbon 18, 281 (1980)

34) J. Zawadski, Carbon 19, 19 (1981)

35) J.S. Mattson, L. Lee, H.B. Mark. Jr. and W.J. Weber, Jr., Colloid Interface Sci. 33, 284 (1970)

36) M. Voll and H.P. Boehm, Carbon 9, 481 (1971)

37) H. Yoshida, Y. Adachi and K. Kamegawa Tanso 1982 [No. 111] 149

38) L.J. Bellamy, The Infra-Red Spectra of Complex Molecules, Third Edition; Chapman and Hall; London (1975) p. 167

39) E. Papirer, S. Li and J.B. Donnet, Carbon 25, 243 (1987) 